\title{
Huge solid pseudopapillary tumor of the pancreas 'Frantz tumor': a case report
}

\author{
Abdullah Saleh AlQattan ${ }^{\wedge}$, Hassan M. Alshaqaq ${ }^{2}$, Alaa A. Al Abdrabalnabi, Muna Alnamlah ${ }^{2}$, \\ Ahmed Abdulmajeed Alanazi ${ }^{1} \wedge$, Mohammed Saad Alqahtani ${ }^{1}$ \\ ${ }^{1}$ Hepatopancreaticobiliary Unit, Department of Surgery, King Fahad Specialist Hospital, Dammam, Kingdom of Saudi Arabia; ${ }^{2}$ College of Medicine, \\ Imam Abdulrahman Bin Faisal University, Dammam, Kingdom of Saudi Arabia \\ Correspondence to: Abdullah Saleh AlQattan. Department of General Surgery, Hepatobiliary unit, Building 7, 2nd floor, King Fahad Specialist \\ Hospital-Dammam, Kingdom of Saudi Arabia. Email: a.qattan.94@gmail.com.
}

\begin{abstract}
Solid pseudopapillary neoplasms (SPN) of the pancreas are rare neoplasms accounting for 1-2\% of all pancreatic tumors and have a general female predominance. We report a case and intraoperative videos of SPN involving the whole pancreatic tail. A 19-year-old female patient initially presented to another healthcare facility complaining of abdominal pain, which was started 6 years ago. A contrast-enhanced Computed Tomography (CT) scan of the abdomen showed a large mass measuring $15.6 \mathrm{~cm} \times 11.6 \mathrm{~cm} \times$ $11 \mathrm{~cm}$, arising from the pancreas with an enhancing cystic component. The patient underwent exploratory laparotomy, which revealed a huge mass occupying most of the abdominal cavity. Thus, we proceeded with a distal pancreatectomy and splenectomy. Intraoperatively, the frozen section showed that the mass had features of a solid pseudopapillary tumor of the pancreas with negative resection margins. The SPN diagnosis was confirmed by histopathology and immunohistochemistry. The pathophysiology behind the development of SPN and its cellular origin is still a matter of debate with multiple proposed hypotheses. SPNs are asymptomatic in almost $70 \%$ of all cases and usually discovered incidentally. The pre-operative diagnosis of SPNs remains a clinical challenge despite all the current advances in the diagnostic modalities. Surgical management with negative resection margins is the mainstay of treatment, even with metastasis and vascular invasion, surgical excision should be performed whenever feasible. The recurrence rate after surgical resection has been reported to be 3-9\%. The prognosis of SPN limited to the pancreas is generally excellent with over $95 \%$ cure rate following complete surgical resection. SPN is a rare entity of a controversial origin but is considered as a low-grade malignancy. Surgical resection to achieve complete excision constitutes the mainstay of treatment, which mostly results in an excellent prognosis.
\end{abstract}

Keywords: Pseudopapillary; neoplasms; Frantz; pancreas; case report; pancreatectomy

Submitted Apr 10, 2020. Accepted for publication Jul 31, 2020.

doi: 10.21037/jgo-20-180

View this article at: http://dx.doi.org/10.21037/jgo-20-180

\section{Introduction}

Solid pseudopapillary neoplasms (SPN) of the pancreas, also known as 'Frantz tumor' attributed to Virginia K. Frantz, who was the first to describe it in the 1950s. SPN had many different names until its official nomenclature by the World
Health Organization (WHO) in $1996(1,2)$. However, it was not recognized by the $\mathrm{WHO}$ as a low-grade epithelial malignant neoplasm until $2010(1,2)$. It was reported to have an incidence of $1-2 \%$ of all pancreatic tumors with a favorable prognosis after surgical excision (3). SPNs are

^ ORCID: Abdullah Saleh AlQattan, 0000-0002-2101-9945; Hassan M. Alshaqaq, 0000-0003-4702-0917; Alaa A. Al Abdrabalnabi, 00000002-6860-0438; Muna Alnamlah, 0000-0003-2903-8371; Ahmed Abdulmajeed Alanazi, 0000-0002-8035-2449. 

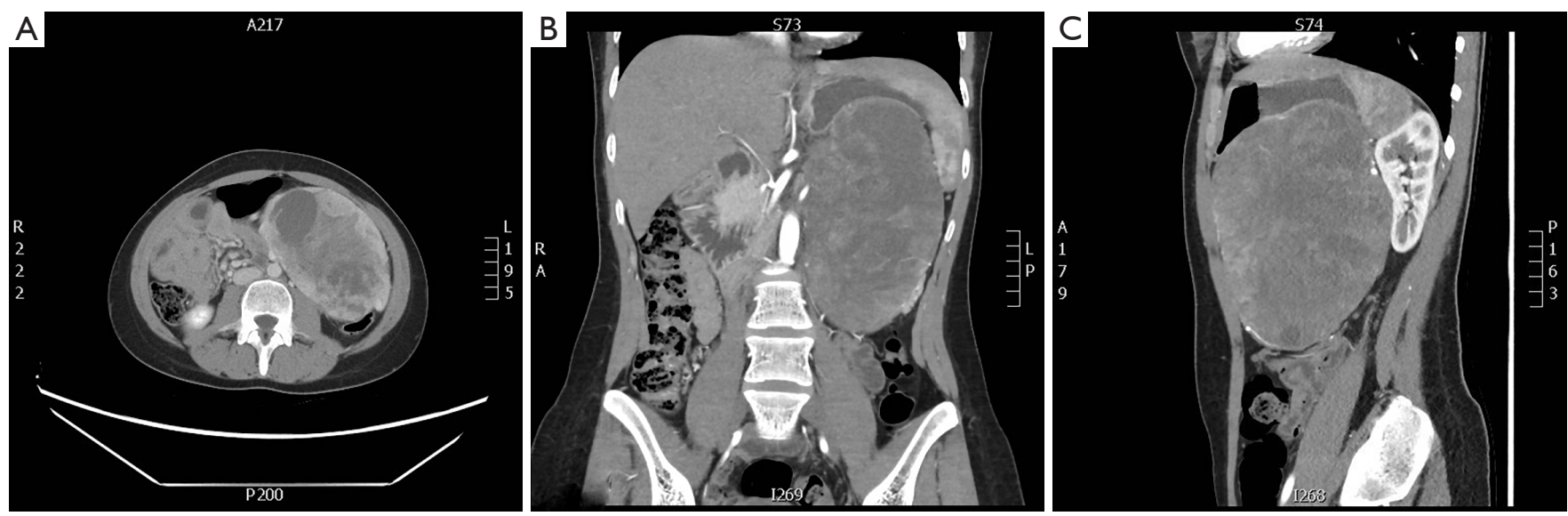

Figure 1 A contrast-enhanced computed tomography scans of the abdomen showing a large, well-defined, and heterogeneous tumor measuring $15.6 \mathrm{~cm} \times 11.6 \mathrm{~cm} \times 11 \mathrm{~cm}$, arising from the pancreas with an enhancing cystic component.

usually encountered in young adult females, with a female to male ratio of 11 to 1 ; nevertheless, a small percentage occurs in the pediatric age group (4-6). The tumor location tends to be in the body or tail of the pancreas in $60 \%$ of the cases, while the remaining are located in the head of the pancreas $(1,6,7)$. Even though SPNs are labeled as low malignant tumors, there have been multiple reports of metastasis $(6,8,9)$, with an estimated incidence reaching up to $10 \%$ of the cases $(1,2)$. We report the following case in accordance with the CARE reporting checklist (available at http://dx.doi.org/10.21037/jgo-20-180).

\section{Case presentation}

A 19-year-old female patient not known to have any medical illnesses was referred to our surgical clinic after she initially presented to another healthcare facility complaining of abdominal pain, which was started 6 years ago. It was sharp epigastric pain radiating to the back. The pain was aggravated by lying supine and alleviated by leaning forward. The patient also noticed abdominal distention. She denied any history of vomiting, change in bowel habits, weight loss, night sweats, pruritis, scleral or urinary discoloration, and menstrual irregularities. Surgical and family history of pancreatic diseases were deemed negative. During the year before her presentation, she experienced early satiety, increased abdominal pain, and abdominal distention along with weight loss of $10 \mathrm{~kg}$. An ultrasound was performed in the referring facility and showed a heterogeneously hypoechoic irregular mass that is originating from the distal pancreas.
In our hospital, her abdominal examination showed: a soft, lax, non-tender abdomen with a palpable mass occupying most of her abdomen. All of her laboratory work-up, including tumor markers, were within normal limits.

A contrast-enhanced CT scan of the abdomen (Figure 1) showed a large mass measuring $15.6 \mathrm{~cm} \times 11.6 \mathrm{~cm} \times 11 \mathrm{~cm}$, arising from the pancreas with an enhancing cystic component. The visualized part of the pancreatic duct was not dilated. The mass was compressing the stomach and jejunal loops, pushing them superiorly and towards the right side of the abdomen, respectively. The mass was also compressing the left kidney, left renal, and the superior mesenteric veins with no evidence of thrombosis.

Furthermore, there was no evidence of retroperitoneal lymph nodes involvement. Hence, the decision to perform distal pancreatectomy and splenectomy was made. Two weeks prior to her surgery, the patient was given pneumococcal, haemophilus influenzae, and meningococcal vaccines.

The patient underwent exploratory laparotomy, which revealed a huge mass occupying most of the abdominal cavity and displacing the stomach superiorly. Subsequently, we proceeded with a distal pancreatectomy and splenectomy (Videos 1,2,3). Intraoperatively, the frozen section showed that the mass had features of a solid pseudopapillary tumor of the pancreas with negative resection margins. The gross examination of the mass revealed an: encapsulated, heterogeneous mass with both cystic and solid components involving the whole pancreatic tail with no local invasion to the spleen (Figure 2). 

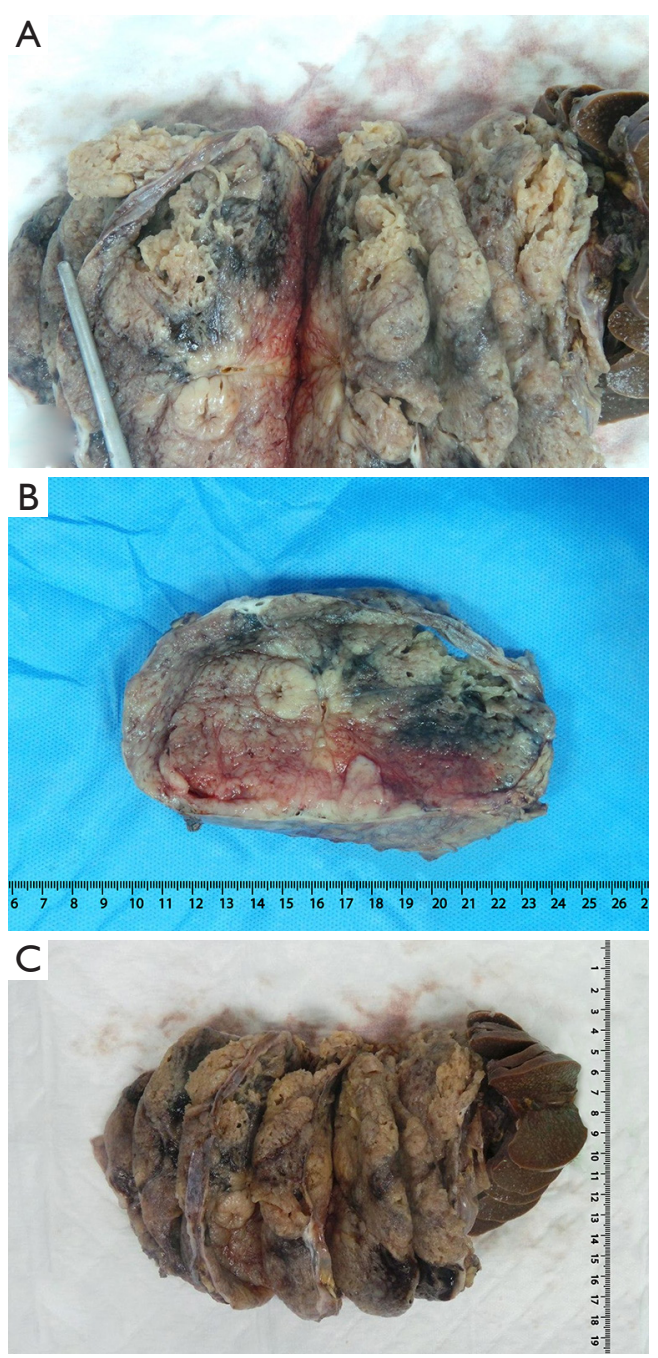

Figure 2 Gross examination of the resected mass showing an encapsulated, heterogeneous mass with both cystic and solid components.

Histopathological examination of the surgical specimen (H\&E staining) demonstrated a pseudopapillary architecture (Figure $3 A$ ). Immunohistochemical staining showed the tumor being diffusely positive for progesterone receptor (Figure 3B), synaptophysin (Figure 3C), beta-catenin (Figure $3 D$ ), and CD10 (Figure $3 E$ ). In contrast, it was negative for chromogranin (Figure $3 F$ ), and estrogenic receptor. The Ki-67 was $1-2 \%$. Consequently, the diagnosis of a solid pseudopapillary tumor was confirmed with negative resection margins. Moreover, no lymph node involvement was found out of the fourteen examined lymph nodes, and no lymphovascular or perineural invasion was identified.
The spleen was unremarkable (Pathological TNM $=\mathrm{T}: 3$ $\mathrm{pN}: 0 \mathrm{M}: \mathrm{x})$. The patient was discharged after a smooth and uneventful postoperative course. The patient is following up in which she showed neither signs of recurrence nor the emergence of metastatic lesions 1 year postoperatively.

All procedures performed in studies involving human participants were in accordance with the ethical standards of the institutional and/or national research committee(s) and with the Helsinki Declaration (as revised in 2013). Written informed consent was obtained for publication of this case report and accompanying images. A copy of the written consent is available for review by the Editor-in-Chief of the journal on request.

\section{Discussion}

SPNs account for a small percentage of all pancreatic tumors ranging from $0.17 \%$ to $2.7 \%$ ( 7 ). The pathophysiology behind the development of SPN and its cellular origin is still a matter of debate with multiple proposed hypotheses in the literature $(5,10,11)$. One of the theories that has been suggested is the role of sex hormones in the pathogenesis of this tumor $(5,11-16)$. It was proposed based on the higher female predominance rate that has been reported, particularly during the reproductive age, along with the fact that SPNs are usually positive for these receptors (5,11-16). It is supported by the fact that during the embryological stage, the genital ridges are located adjacent to the pancreatic anlage (17).

Another hypothesis attributes the development of SPNs to different genetic mutations, and the most studied is the nuclear expression of b-catenin and vimentin secondary to genetic mutation and the interference with the Wnt signaling pathway, which has been reported to be present in up to $90 \%$ of these tumors $(5,6)$. The role of other genetic mutations like the p53 gene and $\mathrm{k}$-ras has been studied, but no evidence of their involvement was found $(5,6)$.

Furthermore, since SPNs also show some focal positivity for other non-sex hormonal markers. The idea of focal neuroendocrine differentiation was suggested (10). One of the factors that might have a role in the development of SPNs is viral hepatitis, namely hepatitis B and C viruses as it has been reported in the literature to be associated with SPNs in up to $62 \%$ of patients, but this has not been well established (6).

Although, $70 \%$ of all cases are symptomatic, SPNs are discovered incidentally in $30 \%$ of the cases (17). Once the tumor grows and becomes large enough to cause pressure 

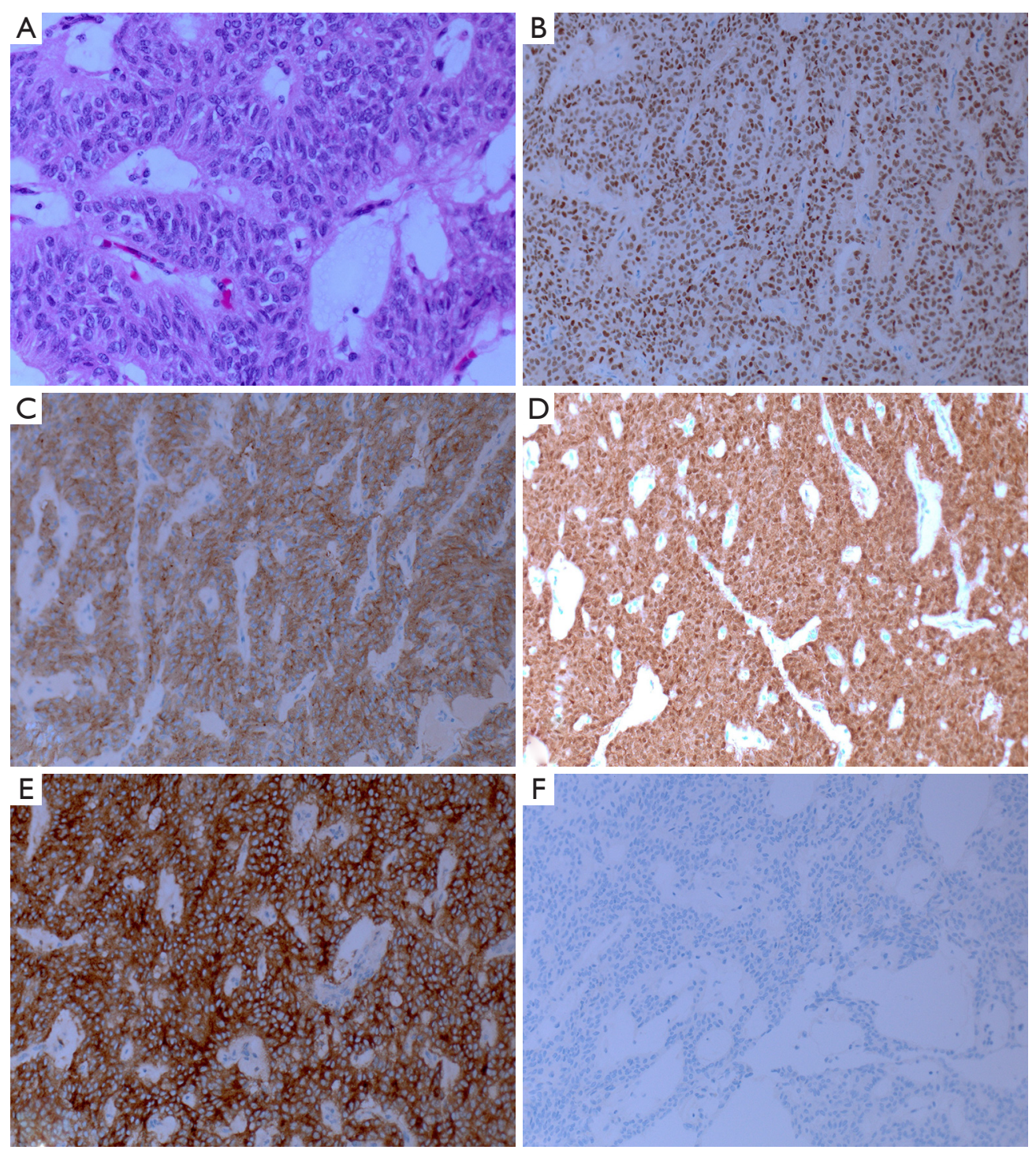

Figure 3 Histopathological examination of the surgical specimen $(\times 20)$. H\&E staining (A) demonstrating a pseudopapillary architecture; immunohistochemical study showing the tumor being diffusely positive for progesterone receptor (B), synaptophysin (C), beta-catenin (D), and CD10 (E), but negative for chromogranin (F).

symptoms to the adjacent organs, most patients complain of abdominal pain, followed by an increase in abdominal girth $(1,17)$. That was evident in our case as the patient had an on-and-off abdominal pain for years, then started to have early satiety and a noticeably increased abdominal girth.

Even though SPNs are slow-growing tumors with a low Ki-67 index, several case reports have shown different growth rates in terms of doubling time starting from 240 to 765 days (18-20). This wide range suggests that although these are collectively slow-growing neoplasms; however, the growth rate varies significantly (19). Despite the unclear pathogenesis and source of the tumor (21), the authors believe that this huge tumor size potentially resulted due to the delayed presentation that lasts for six years.

One of the rare presentations of SPNs is tumor rupture that is commonly seen after blunt abdominal trauma, which has been reported to represent $8 \%$ of the cases $(17,22)$. Interestingly, $\mathrm{Xu}$ et al. reported a spontaneous rupture of SPN in a 22 years old female who presented to the Emergency Department (ED) complaining of abdominal pain associated with leukocytosis and hemoglobin drop secondary to spontaneous SPN rupture and bleeding (22). 
The suggested theory of spontaneous rupture is that the cystic part of the SPN undergoes internal degeneration with a subsequent hemorrhage; consequently, if the bleeding was significant enough, it can lead to increased intramural pressure causing the spontaneous rupture $(17,22)$.

The pre-operative diagnosis of SPNs remains a clinical challenge despite all the current advances in diagnostic modalities (9). This is due to the overlap with a wide range of differential diagnoses such as benign cystic lesions, including pseudocysts, hydatid cysts, cystadenoma, lymphangioma, and hemangioma; as well as, different malignant lesions such as; cystadenocarcinoma or intraductal papillary mucinous neoplasms $(5,7)$. When it comes to the pediatric age group, pancreatic tumors of secondary origin like neuroblastoma, leukemia, lymphoma, and lymphoproliferative disorders are more common (5).

Tumor markers like alfa fetoprotein, carcinoembryonic antigen, CA199, CA125, and CA242 might be elevated, but they are not specific for SPNs (6). Nevertheless, these tumor markers and pancreatic tumor markers should be considered during the work-up as other malignant tumors are still part of the differential diagnosis of SPNs (9).

Imaging wise, abdominal CT scan with intravenous contrast has been reported to be the best imaging modality because it provides not only the origin, size, and layout of the tumor but also the presence of local invasion and metastasis (5). As SPNs have a mix of both solid and cystic components, areas of enhancing and non-enhancing lesions are seen and are surrounded by a capsule along with intratumoral calcifications (5). Furthermore, hemorrhage may result due to the growth of the tumor and subsequent internal degeneration (22). The presence of an encapsulated mass consisting of both cystic and solid components and intratumoral hemorrhage are useful factors to distinguish SPNs from its other malignant differentials $(5,10,22)$. With the presence of these pathognomonic features of SPNs, a CT scan is considered adequate to establish the preoperative diagnosis $(23,24)$.

On the other hand, Magnetic Resonance Imaging (MRI), is considered as a second-line imaging modality as it can demonstrate further information with regards to hemorrhage and necrosis of the tumor's tissue $(23,24)$. Typically, SPNs would show a vascular, encapsulated mass composed of both mixed cystic and solid components with a high-signal intensity on $\mathrm{T} 1$ and low signal intensity on T2 series representing hemorrhagic areas on MRI $(22,25)$. Dan et al. have highlighted that MRI was not necessary in their reported cases of SPNs located in the tail of the pancreas, where a CT scan was able to demonstrate the pathognomonic features of SPN $(23,24)$.

A pre-operative histopathologic diagnosis could be achieved by endoscopic ultrasound (EUS) biopsy (26). Still, its use has the downside of seeding of tumor cells into the peritoneum or the gastric wall, which has been reported in multiple cases of pancreatic adenocarcinoma $(7,26)$. Yamaguchi et al. have reported the seeding of SPN cells into the gastric wall with a subsequent tumor formation secondary to EUS biopsy 67 months after the procedure (26).

Hanada et al. conducted a nationwide, multicentric, retrospective, and questionnaire-based survey study across Japan to assess the clinicopathological features of SPNs (27). The study included 288 patients who were diagnosed with SPN between January 1990 and March 2015 (27). They have evaluated the capability of using a single imaging modality to establish a pre-operative diagnosis, which ranged between $50 \%$ to $70 \%$. Additionally, the detection of the cystic component was higher on both MRI and EUS compared to CT scan, while the detection of calcifications on CT scan and EUS was of similar rate (27). Hence, the recommendation was to use a combination of imaging modalities in order to establish a pre-operative diagnosis (27). In our case, due to the typical clinical presentation, along with the presence of the pathognomonic features on CT scan and US, the need for further imaging was waived.

Surgical management with free surgical resection margins is the mainstay of treatment even with metastasis and vascular invasion, surgical excision should be performed whenever feasible (9). Radical lymphadenectomy is not indicated in these cases (5). The recurrence rate after surgical resection has been reported to be 3-9\% (10). Regardless, patients should be promptly followed up due to the risk of potential recurrence or emergence of metastatic lesions (20). Even in case of recurrence or metastasis, surgery remains the treatment of choice. However, in unresectable lesions, surgical debulking might be justified $(7,9)$.

There are two types of resection, depending on the location of the tumor. When it is located in the body or tail of the pancreas, distal pancreatectomy with or without splenic preservation should be performed (5). On the other hand, pancreaticoduodenectomy is performed if the tumor is located in the head of the pancreas (5). The surgical resection must be performed with caution to avoid rupture or spillage of the tumor content, which can result in the seeding of the tumor cells into the peritoneum $(8,26)$. Due to the encapsulation and low malignant potential of SPNs, 
it has been advocated to perform the surgical management as conservatively as possible (5).

Even though SPN has a low malignant potential, local invasion and metastasis have been reported (10). Moreover, major sites affected were the liver and peritoneal cavity $(10,22)$. Nevertheless, the outcome is encouraging, as longterm survival in patients with liver metastasis showed better chances when an excisional resection was performed (5). In addition to the radiosensitivity characteristic of SPN, chemotherapy with metastatic liver lesions seems to have a valuable role (5). However, both modalities are still under scrutiny and only considered as an alternative in the case of surgical contraindications $(5,20)$. Besides, extrapancreatic SPNs coexisted in $0.62 \%$ of reported cases, and are frequently seen with either testicular or ovarian origin (6).

Although SPN grows largely with features of invasion, nearly all patients who received complete surgical excision have demonstrated an excellent chance for long-term survival (5). The prognosis of SPN limited to the pancreas is generally excellent, with over $95 \%$ cure rate following complete surgical resection (5). Furthermore, local invasion and metastasis are not considered contraindications for surgical resection, and even patients with unresectable tumors can survive for more than ten years post-surgical debulking $(5,10)$.

\section{Conclusions}

SPN is a rare entity of a controversial origin but is considered to be of a low-grade malignancy and a favourable prognosis. It shows strong female predominance with unspecified presentation. Imaging modalities aid to differentiate this entity from other malignancies; however, postoperative histopathological examination and immunohistochemistry remain the primary diagnostic tools. Surgical resection to achieve complete excision constitutes the mainstay of treatment and mostly results in an excellent prognosis. The survival benefit of repeated surgical resection for recurrence is encouraging.

\section{Acknowledgments}

Funding: None.

\section{Footnote}

Reporting Checklist: The authors presented the case report in accordance with the CARE reporting checklist. Available at http://dx.doi.org/10.21037/jgo-20-180
Peer Review File: Available at http://dx.doi.org/10.21037/ jgo-20-180

Conflicts of Interest: All authors have completed the ICMJE uniform disclosure form (available at http://dx.doi. org/10.21037/jgo-20-180). The authors declare no conflict of interest.

Ethical Statement: The authors are accountable for all aspects of the work in ensuring that questions related to the accuracy or integrity of any part of the work are appropriately investigated and resolved. All procedures performed in studies involving human participants were in accordance with the ethical standards of the institutional and/or national research committee(s) and with the Helsinki Declaration (as revised in 2013). Written informed consent was obtained for publication of this case report and accompanying images. A copy of the written consent is available for review by the Editor-in-Chief of the journal on request.

Open Access Statement: This is an Open Access article distributed in accordance with the Creative Commons Attribution-NonCommercial-NoDerivs 4.0 International License (CC BY-NC-ND 4.0), which permits the noncommercial replication and distribution of the article with the strict proviso that no changes or edits are made and the original work is properly cited (including links to both the formal publication through the relevant DOI and the license). See: https://creativecommons.org/licenses/by-nc-nd/4.0/.

\section{References}

1. Torres OJM, Rezende MB De, Waechter FL, et al. Pancreatoduodenectomy for solid pseudopapillary tumor of the pancreas: A multi-institution study. Arq Bras Cir Dig 2019;32:e1442.

2. Tan HL, Tan EK, Teo JY, et al. Outcome of minimallyinvasive versus open pancreatectomies for solid pseudopapillary neoplasms of the pancreas: A 2:1 matched case-control study. Ann Hepatobiliary Pancreat Surg 2019;23:252.

3. Tsujie M, Wakasa T, Mizuno S, et al. Solid pseudopapillary neoplasm of the pancreas showing marked distal atrophy: A case report. Int J Surg Case Rep 2019;55:136-9.

4. Song H, Dong M, Zhou J, et al. Solid Pseudopapillary Neoplasm of the Pancreas: Clinicopathologic Feature, Risk Factors of Malignancy, and Survival Analysis of 53 Cases from a Single Center. Biomed Res Int 2017;2017:5465261. 
5. Papavramidis T, Papavramidis S. Solid pseudopapillary tumors of the pancreas: Review of 718 patients reported in english literature. J Am Coll Surg 2005;200:965-72.

6. Gurzu S, Bara T, Sincu M, et al. Solid pseudopapillary neoplasm of pancreas: Two case reports. Medicine (Baltimore) 2019;98:e16455.

7. Branco C, Vilaça S, Falcão J. Solid pseudopapillary neoplasm-Case report of a rare pancreatic tumor. Int J Surg Case Rep 2017;33:148-50.

8. Wu H, Huang YF, Liu XH, et al. Extrapancreatic solid pseudopapillary neoplasm followed by multiple metastases: Case report. World J Gastrointest Oncol 2017;9:497-501.

9. Chon HK, Choi KH, Kim TH. An Unusual Presentation of a Solid Pseudopapillary Tumor of the Pancreas Mimicking Adenocarcinoma. Clin Endosc 2020;53:615-9.

10. Sharma PK, Mehrotra S, Gleisner AL, et al. Recurrent Solid Pseudopapillary Neoplasm of Pancreas: Case Report and Review of Literature. J Pancreat Cancer 2018;4:25-9.

11. Cuccurullo D, Carbone G, Iovino MG, et al. pancreatic pseudopapillary tumor managed laparoscopically: A case report and review of the literature. Int J Surg Case Rep 2018;45:4-8.

12. Wrba F, Chott A, Ludvik B, et al. Solid and cystic tumor of the pancreas; a hormonal-dependent neoplasm? Histopathology 1988;12:338-40.

13. Lam KY, Lo CY, Fan ST. Pancreatic solid-cystic-papillary tumor: Clinicopathologic features in eight patients from Hong Kong and review of the literature. World J Surg 1999;23:1045-50.

14. Nishihara K, Tsuneyoshi M, Ohshima A, et al. Papillary Cystic Tumor of the Pancreas: Is it a Hormone-dependent Neoplasm? Pathol Res Pract 1993;189:521-6.

15. Zamboni G, Bonetti F, Scarpa A, et al. Expression of progesterone receptors in solid-cystic tumour of the pancreas - A clinicopathological and immunohistochemical study of ten cases. Virchows Arch A Pathol Anat Histopathol 1993;423:425-31.

16. Lee WY, Tzeng CC, Chen RM, et al. Papillary cystic tumors of the pancreas: assessment of malignant potential by analysis of progesterone receptor, flow cytometry, and ras oncogene mutation. Anticancer Res 1997;17:2587-91.

17. Mirminachi B, Farrokhzad S, Sharifi AH, et al. Solid Pseudopapillary Neoplasm of Pancreas; A Case Series and Review Literature. Middle East J Dig Dis 2016;8:102-8.

18. Asari Y, Shimazu S, Nishimura H, et al. A Case Study of Giant Solid and Cystic Tumor of the Pancreas. Japanese J Gastroenterol Surg 1991;24:2461-5.

19. Kato T, Egawa N, Kamisawa T, et al. A case of solid pseudopapillary neoplasm of the pancreas and tumor doubling time. Pancreatology 2002;2:495-8.

20. Sperti C, Berselli M, Pasquali C, et al. Aggressive behaviour of solid-pseudopapillary tumor of the pancreas in adults: A case report and review of the literature. World J Gastroenterol 2008;14:960-5.

21. Cuccurullo D, Carbone G, Iovino MG, et al. Solid pancreatic pseudopapillary tumor managed laparoscopically: A case report and review of the literature. Int J Surg Case Rep 2018;45:4-8.

22. Xu X, Chen D, Cao L, et al. Spontaneous rupture of solid pseudopapillary tumor of pancreas: A case report and review of literature. Medicine (Baltimore) 2019;98:e17554.

23. Rajtar KZ, Sznajder K, Milto KM. Diagnostic imaging of a solid pseudopapillary tumour of the pancreas in a 20-year-old woman - A case study. Prz Gastroenterol 2016;11:214-7.

24. Dan D, Rambally R, Cawich SO, et al. Solid pseudopapillary neoplasms of the pancreas: a report of two cases. Case Rep Med 2014;2014:356379.

25. Hansen CP, Kristensen TS, Storkholm JH, et al. Solid pseudopapillary neoplasm of the pancreas: Clinicalpathological features and management, a single-center experience. Rare Tumors 2019;11:2036361319878513.

26. Yamaguchi H, Morisaka H, Sano K, et al. Seeding of a Tumor in the Gastric Wall after Endoscopic Ultrasoundguided Fine-needle Aspiration of Solid Pseudopapillary Neoplasm of the Pancreas. Intern Med 2020;59:779-82.

27. Hanada K, Kurihara K, Itoi T, et al. Clinical and Pathological Features of Solid Pseudopapillary Neoplasms of the Pancreas: A Nationwide Multicenter Study in Japan. Pancreas 2018;47:1019-26.
Cite this article as: AlQattan AS, Alshaqaq HM, Al Abdrabalnabi AA, Alnamlah M, Alanazi AA, Alqahtani MS. Huge solid pseudopapillary tumor of the pancreas 'Frantz tumor': a case report. J Gastrointest Oncol 2020;11(5):1098-1104. doi: 10.21037/jgo-20-180 